\title{
ON THE COHOMOLOGY OF CHEVALLEY GROUPS ${ }^{1}$
}

\author{
BY ERIC M. FRIEDLANDER AND BRIAN PARSHALL
}

Let $G$ be a simple, simply connected algebraic group defined and split over the prime field $F_{p}, p \geqslant 3$. Let $V$ be a finite dimensional rational $G$-module. As shown in [1], for $d$ suitably large, the Eilenberg-Mac Lane cohomology groups $H^{*}\left(G\left(F_{p} d\right), V\right)$ achieve a stable value $H_{\text {gen }}^{*}(G, V)$, the so-called generic cohomology of $V$. This generic cohomology can in turn be determined from the rational cohomology groups $H_{\text {rat }}^{*}\left(G, V^{(r)}\right), r \geqslant 1$, where $V^{(r)}$ stands for the module $V$ with the action of $G$ twisted by the $r$ th power of the Frobenius morphism $\sigma: G \rightarrow G$. In this paper we announce an explicit determination of the cohomology groups $H_{\text {rat }}^{*}\left(G, V^{(r)}\right)$, and hence of the generic cohomology groups $H_{\text {gen }}^{*}(G, V)$, in a range of cohomology degrees (restricted by the prime $p$ ) for an arbitrary irreducible rational module $V$ whose high weight lies in the bottom $p$-alcove. In particular, we obtain stability in $H_{\text {gen }}^{n}(G, V)$ for large $p$, answering a question raised by Scott [6]. Our methods involve a determination in a range of degrees of the cohomology of the restricted enveloping algebra of the Lie algebra of $G$.

The general Theorem 4 below is motivated by the "experimental evidence" provided by Theorem 1. This result concerning the general linear group is particularly strong in terms of its range of degrees, its applicability to small fields, and its description of the $k$-algebra structure. Here $k$ is an algebraically closed field of characteristic $p$.

THEOREM 1. The following $k$-algebras are isomorphic in degrees less than $\min (2 p-1, d(2 p-3)-2)$, where $q=p^{d}$ :

$$
H^{*}\left(G L_{n}\left(F_{q}\right), M_{n}\right) ; \quad H_{\mathrm{rat}}^{*}\left(G L_{n}, M_{n}^{(r)}\right), \quad r \geqslant 1 ; \quad k[t] /\left(t^{n}\right), \operatorname{deg}(t)=2 .
$$

In Theorem $1, M_{n}$ is viewed as the adjoint representation of the algebraic group $G L_{n}$ over $k$. We emphasize that $H^{*}\left(G L_{n}\left(F_{q}\right), M_{n}\right)$ and $H_{\text {rat }}^{*}\left(G L_{n}, M_{n}^{(r)}\right)$ have natural $k$-algebra structures because $G L_{n}$ acts as (associative) $k$-algebra automorphisms on $M_{n}$. These multiplicative structures are analyzed using external comultiplications in cohomology induced by external tensor product maps $G L_{m} \times G L_{n} \rightarrow G L_{m n}$.

The key step in the proof of Theorem 1 is the identification of

$$
H^{*}\left(B_{n}\left(F_{q}\right), M_{n}\right)
$$

Received by the editors November 30, 1981.

1980 Mathematics Subject Classification. Primary 20J06, 20G10, 14 L17.

1 Research supported by NSF. 
in the appropriate range of degrees, where $B_{n}\left(F_{q}\right)$ is the Borel subgroup of upper triangular matrices in $G L_{n}\left(F_{q}\right)$. This is achieved by studying the spectral sequences associated to the extension

$$
1 \rightarrow R_{n}\left(F_{q}\right) \rightarrow B_{n}\left(F_{q}\right) \rightarrow B_{n-1}\left(F_{q}\right) \rightarrow 1
$$

and an appropriate filtration of the $B_{n}\left(F_{q}\right)$-module $M_{n}$.

Theorem 1 has the following two corollaries. This first is based on the work of [2], which inspired the present study.

CoROllaRY 2. For $p \geqslant 5, K_{5}\left(Z / p^{2} Z\right)$ has nonzero p-torsion.

Corollary 3. Let $h: G L_{n} \rightarrow G$ be the hyperbolic map, where $G=S O_{2 n}$, $\mathrm{SO}_{2 n+1}$, or $S p_{2 n}$. Then there is an induced map

$$
h^{*}: H^{i}\left(G\left(F_{q}\right), \mathfrak{g}\right) \rightarrow H^{i}\left(G L_{n}\left(F_{q}\right), M_{n}\right)
$$

which is surjective for $i=4 j-2<\min (2 p-2, d(2 p-3)-2)$, where $g$ is the adjoint representation of $G$.

We now consider a simple, simply connected algebraic group $G$ defined and split over $F_{p}$ and an irreducible module $V_{\lambda}$ with highest weight $\lambda$ in the closure of the bottom $p$-alcove of the weight lattice of $G$. Such a $G$-module is obtained by reducing modulo $p$ the complex irreducible module $V(\lambda)$ of highest weight $\lambda$ for the complex Lie algebra $g_{\mathrm{C}}$ associated to $G$.

THEOREM 4. Let $G$ be as described above, and assume also that $p>2 h$, where $h$ is the Coxeter number of the root system of $G$. Let $\lambda$ be a dominant weight in the closure of the bottom p-alcove of the weight lattice of $G$. For any $r \geqslant 1$, $H_{\mathrm{rat}}^{i}\left(G, V^{(r)}\right)$ has dimension equal to the number of "generalized exponents" of $\lambda$ which equal $i / 2$, provided that $i$ is less than $1 / 4(p-2 h+2)$.

Following Hesselink [3], generalized exponents $m_{1}(\lambda) \leqslant \cdots \leqslant m_{l_{\lambda}}(\lambda)$ of a dominant weight $\lambda$ can be determined as follows. Let $\Phi^{+}$be the set of positive roots in the root system $\Phi$ of $g_{C}, \rho=1 / 2 \Sigma_{\alpha \in \Phi^{+}} \alpha$. For a weight $\chi$ let $p_{n}(\chi)$ be the number of maps $f$ from $\Phi^{+}$into the nonnegative integers satisfying $n=$ $\Sigma f(\alpha)$ and $\chi=\Sigma f(\alpha) \alpha$. If $W$ is the Weyl group, define

$$
d_{n}(\lambda)=\sum_{w \in W} \operatorname{det}(w) p_{n}(w(\lambda+\rho)-\rho)
$$

Then $m_{i}(\lambda)=\min \left\{m: i \leqslant d_{0}(\lambda)+\cdots+d_{m}(\lambda)\right\}$. The generalized exponents arise in describing the $G_{\mathrm{C}}$-module structure of the algebra of polynomial functions on $g_{C}$ (Kostant [4]). For $\lambda$ the high weight of the adjoint representation, they are the usual exponents. 
As a corollary of Theorem 4 and the results of [1], we conclude the following result:

Corollary 5. Assume the notation of Theorem 4, and let $i$ be in the range indicated there. Then $H_{\mathrm{gen}}^{i}\left(G, V_{\lambda}\right) \cong H_{\mathrm{rat}}^{i}\left(G, V_{\lambda}^{(r)}\right), r \geqslant 1$. Consequently, $H_{\mathrm{gen}}^{i}\left(G, V_{\lambda}\right)$ has dimension equal to the number of generalized exponents of $\lambda$ equal to $i / 2$.

We remark that the arithmetic results of [1] give conditions on $d$ which guarantee that $H^{i}\left(G\left(F_{p} d\right), V_{\lambda}\right) \cong H_{\text {gen }}^{i}\left(G, V_{\lambda}\right)$.

The proof of Theorem 4 proceeds with an analysis of the infinitesimal extension of affine $k$-groups

$$
1 \rightarrow G_{r} \rightarrow G \stackrel{\sigma^{r}}{\rightarrow} G \rightarrow 1 .
$$

Induction reduces the analysis to the case $r=1$, in which case $G_{1}$ is the infinitesimal group whose associated finite dimensional Hopf algebra has dual isomorphic to the restricted enveloping algebra $V(\mathfrak{g})$ of the Lie algebra $\mathfrak{g}$ of $G$. The cohomology groups $H^{*}(V(\mathrm{~g}), k)$ when viewed as rational representations of $G / G_{1}$ are duals of Weyl modules in a range of degrees. The computation of $H_{\mathrm{rat}}^{*}\left(G, V_{\lambda}^{(r)}\right)$ is then reduced, in view of results of [1], to the following fundamental result which was motivated by May [5]:

THEOREM 6. Let $G$ be a simple, simply connected algebraic group defined and split over $F_{p}$ with Lie algebra $\mathfrak{g}$. There exists a natural first quadrant spectral sequence of differential graded algebras

$$
E_{0}^{m, m+n}=S^{m}\left(\mathfrak{g}^{\#}\right)^{(1)} \otimes \Lambda^{n}\left(\mathrm{~g}^{\#}\right) \Rightarrow H^{2 m+n}(V(\mathfrak{g}), k)
$$

whose differentials are G-invariant derivations, where $\mathfrak{g}^{\#}$ is the $k$-dual of $\mathrm{g}$. This implies the isomorphism of G-modules

$$
H^{i}(V(\mathfrak{g}), k) \cong A^{i / 2}\left(\mathrm{~g}^{\#}\right)^{(1)}
$$

in a range (given in Theorem 4), where $A^{*}\left(g^{\#}\right)$ is the space of "harmonics" determined by a G-isomorphism (in a range) $A^{*}\left(\mathfrak{g}^{\#}\right) \otimes S^{*}\left(\mathfrak{g}^{\#}\right)^{G} \cong S^{*}\left(\mathfrak{g}^{\#}\right)$.

In view of recent results of Wang [7] on filtrations of tensor products of Weyl modules, the proof of Theorem 4 also applies to provide a computation of generic Ext-groups.

\section{REFERENCES}

1. E. Cline, B. Parshall, L. Scott, and W. van der Kallen, Rational and generic cohomology, Invent. Math. 39 (1977), 143-163.

2. L. Evens and E. M. Friedlander, On $K_{*}\left(Z / p^{2} Z\right)$ and related homology groups, Trans. Amer. Math. Soc. 270 (1982), 1-46.

3. W. Hesselink, Characters of the nullcone, Math. Ann. 252 (1980), 179-182.

4. B. Kostant, Lie group representations on polynomial rings, Amer. J. Math. 85 (1963), 327-404. 
5. J. P. May, The cohomology of restricted Lie algebras and of Hopf algebras, J. Algebra 3 (1966), 123-146.

6. L. Scott, Representations in characteristic p, Proc. Sympos. Pure Math., Vol. 37, Amer. Math. Soc., Providence, R. I., 1980, pp. 319-331.

7. Wang Jian-pan, Sheaf cohomology on $G / B$ and tensor products of Weyl modules, J. Algebra (to appear).

DEPARTMENT OF MATHEMATICS, NORTHWESTERN UNIVERSITY, EVANSTON, ILLINOIS 60201

DEPARTMENT OF MATHEMATICS, UNIVERSITY OF VIRGINIA, CHARLOTTESVILLE, VIRGINIA 22903 